\title{
SÍNCOPE NEUROCARDIOGÊNICA EM CÃO COM DOENÇA VALVULAR CRÔNICA DE MITRAL - RELATO DE CASO
}

\author{
Karina Preising Aptekmann ${ }^{1}$ \\ Rayane Bifano de Oliveira Silva ${ }^{2}$ \\ Afonso Cassa Reis ${ }^{3}$ \\ Paulo Sérgio Cruz de Andrade Júnior ${ }^{4}$ \\ Leandro Egert ${ }^{3}$ \\ Mayara Cristini Ferreira de Aguiar $^{3}$
}

\begin{abstract}
APTEKMANN, K. P.; SILVA, R. B. de O.; REIS, A. F.; ANDRADE JÚNIOR, P. S. C. de; EGERT, L.; AGUIAR, M. C. F. de. Síncope neurocardiogênica em cão com doença valvular crônica de mitral - relato de caso. Arq. Ciênc. Vet. Zool. UNIPAR, Umuarama, v. 19, n. 3, p. 171-174, jul./set. 2016.
\end{abstract}

RESUMO: A doença valvular crônica de mitral é a enfermidade cardíaca mais comumente relatada em cães de pequeno e médio porte, adultos ou idosos. A síncope cardíaca é uma manifestação comumente observada, causada por diversos mecanismos. Objetivou-se relatar um caso desta doença em uma cadela da raça Teckel, com 16 anos de idade, que apresentava episódios recorrentes de síncope. Os episódios estavam associados com bradicardia, palidez de mucosas e aumento da pressão intratorácica. Excluindo-se outras causas de síncope, foi realizado o diagnóstico presuntivo de síncope neurocardiogênica, sendo a primeira descrição na medicina veterinária.

PALAVRAS-CHAVE: Cardiologia. Endocardiose. Reflexo de Bezold-Jarisch. Síncope vasovagal.

\section{NEUROCARDIOGENIC SYNCOPE IN A DOG WITH CHRONIC MITRAL VALVULAR DISEASE - CASE REPORT}

\begin{abstract}
Chronic mitral valvular disease is the most commonly acquired heart disease in middle-aged to elderly dogs in small to medium-size breeds. Cardiac syncope is a common manifestation observed in such disease, caused by several mechanisms. The purpose of this study is to report a case of several syncope episodes in a Teckel dog aged 16 years, presenting chronic mitral valvular disease. The episodes were associated with bradycardia, pale mucous membranes and increased intra-thoracic pressure. The presumptive diagnosis of neurocardiogenic syncope was reached by excluding other causes of syncope, which is the first description in veterinary medicine.

KEYWORDS: Bezold-Jarisch reflex. Cardiology. Endocardiosis. Vasovagal syncope.

\section{SÍNCOPE NEUROCARDIOGÉNICO EN PERRO CON ENFERMEDAD VALVULAR MITRAL CRÓNICA - RELATO DE CASO}

RESUMEN: La enfermedad valvular crónica de mitral es la enfermedad cardíaca más comúnmente reportada en perros de pequeño y mediano tamaño, adultos o ancianos. El síncope cardíaco es una manifestación comúnmente observada, causada por varios mecanismos. Esta investigación ha buscado relatar un caso de esta enfermedad en una perra de la raza Teckel, de 16 años de edad, que presentaba episodios recurrentes de síncope. Los episodios estaban asociados con bradicardia, palidez de mucosas y aumento de la presión intratorácica. Se ha excluido otras causas de síncope, se llevó a cabo el diagnóstico presuntivo de síncope neurocardiogénico, siendo la primera descripción en medicina veterinaria.

PALABRAS CLAVE: Cardiología. Endocardiosis. Reflejo de Bezold-Jarisch. Síncope vasovagal.

\section{Introdução}

A doença valvular crônica de mitral (DVCM) é a doença cardíaca adquirida mais comum em cães, que pode originar insuficiência cardíaca congestiva. É frequentemente reportada em cães adultos e idosos, de raças de pequeno e médio porte, sendo detectada em $30 \%$ dos cães com mais de
13 anos de idade (ABBOT, 2008). A síncope pode ser uma manifestação clínica de cães com esta doença. É definida como uma perda súbita e transitória da consciência, com início rápido e curta duração, ocorrendo recuperação espontânea, rápida e completa (MOYA et al., 2009).

Doenças cardíacas podem causar síncope por diminuição do débito cardíaco (DC) e da pressão arterial sistê-

DOI: https://doi.org/10.25110/arqvet.v19i3.2016.6091

${ }^{1}$ Médica veterinária, doutora. Departamento de Medicina Veterinária, Universidade Federal do Espírito Santo, Alto universitário, s/n, Guararema, CEP 29500-000, Alegre, ES, Brasil. E-mail: kapreising@gmail.com. Telefone 28-3552-8645.

${ }^{2}$ Médica veterinária, graduanda. Departamento de Medicina Veterinária, Universidade Federal do Espírito Santo, Alto universitário, s/n, Guararema, CEP 29500-000, Alegre, ES, Brasil. E-mail:rayanemedvet@hotmail.com

${ }^{3}$ Médico(a) veterinário(a), mestrando(a). Departamento de Medicina Veterinária, Universidade Federal do Espírito Santo, Alto universitário, s/n, Guararema, CEP 29500-000, Alegre, ES, Brasil. E-mail: mayara.cristini@gmail.com (Mayara C. F. Aguiar); afonsocassa@hotmail.com (Afonso C. Reis); leandroegt@ gmail.com (Leandro Egert)

${ }^{4}$ Médico veterinário, mestre. Departamento de Medicina Veterinária, Universidade Federal do Espírito Santo, Hospital Veterinário, Distrito de Rive, CEP 29500-000, Alegre, ES, Brasil. E-mail:mvpaulojr@gmail.com 
mica, com consequente diminuição da pressão de perfusão cerebral. A síncope de origem cardíaca está frequentemente associada com estresse ou esforço, uma vez que o coração comprometido não pode aumentar o DC adequadamente para atender ao aumento da demanda de oxigênio (DAVIDOW; PROW; WOODFIELD, 2001).

A síncope neurocardiogênica, também conhecida como síncope vasovagal, reflexa, neuromediada, vasodepressora ou reflexo de Bezold-Jarisch, desencadeia-se por alguns estímulos, os quais causam bradicardia, diminuição do tônus vascular, ou ambos (GANZEBOOM et al., 2006; AZEVEDO; BARBISAN; SILVA, 2009). Na medicina, é relatado que, em pessoas predispostas, alguns estímulos como a dor, a ansiedade e o estresse podem provocar uma resposta exagerada do sistema nervoso autônomo, que conduz a uma estimulação vagal e inibição do sistema simpático, desencadeando bradicardia e relativa perda da vasoconstrição periférica, resultando em hipotensão. A diminuição dos níveis pressóricos causa hipoperfusão cerebral e um comprometimento da consciência, levando à síncope (AZEVEDO; BARBISAN; SILVA, 2009).

Objetivou-se relatar um caso de uma cadela da raça Teckel diagnosticada com DVCM, apresentando supostamente um quadro de síncope neurocardiogênica.

\section{Relato de caso}

Uma cadela da raça Teckel, com 16 anos de idade, foi atendida no Hospital Veterinário da Universidade Federal do Espírito Santo, em fevereiro de 2012, para um check-up. Não apresentava sinais clínicos, porém, ao exame clínico, detectou-se a presença de sopro sistólico em foco mitral de grau V/VI, frequência cardíaca (FC) de 100 bpm, sem outras alterações. Foram realizados exames complementares que revelaram aumento da silhueta cardíaca na radiografia torácica, sem alterações no padrão pulmonar; ritmo sinusal e evidências de sobrecarga atrial e ventricular esquerda no eletrocardiograma (ECG); ao ecocardiograma (ECO) evidenciou-se DVCM e tricúspide com insuficiência de válvulas mitral, tricúspide, pulmonar e aórtica, sem evidências de disfunção sistólica ou diastólica. Não observou-se alterações no Doppler tecidual. A avaliação da pressão arterial (PA) sistólica sistêmica mostrou-se nos limites da normalidade para a espécie $(140 \mathrm{mmHg})$. Diante do quadro apresentado pelo animal foi feito o diagnóstico de doença valvular crônica de mitral e tricúspide. Optou-se pela não realização do tratamento, uma vez que o animal não apresentava sinais clínicos.

Após um ano (fev/2013), o animal apresentou uma crise aguda de dispneia e cianose. Foi tratada de forma emergencial com oxigenioterapia e administração parenteral de furosemida ${ }^{5}$, aminofilina ${ }^{6}$ e butorfanol ${ }^{7}$, conforme recomendado por De Francesco (2013). Os exames complementares revelaram a presença de edema pulmonar e aumento da silhueta cardíaca na radiografia torácica. O ECG revelou a presença de ritmo sinusal com presença de complexos ventriculares prematuros isolados. O ECO encontrava-se estável, comparado ao exame realizado previamente. $\mathrm{O}$ valor da $\mathrm{PA}$ sistólica sistêmica foi de $130 \mathrm{mmHg}$. A cadela apresentou uma melhora substancial no mesmo dia e foi prescrito o tra-

${ }^{5}$ Furosemida solução injetável 10mg/mL, Teuto, Anápolis, GO, Brasil. ${ }^{6}$ Aminofilina solução injetável $24 \mathrm{mg} / \mathrm{mL}$, Hipolabor, Sabará, MG, Brasil.

${ }^{7}$ Torbugesic solução injetável $10 \mathrm{mg} / \mathrm{mL}$, Fort Dodge, Campinas, SP, Brasil. tamento domiciliar com enalapril ${ }^{8}$, espironolactona ${ }^{9}$, omega $3^{10}$, amiodarona ${ }^{11}$ e furosemida ${ }^{12}$ por período indeterminado. A furosemida foi suspensa após uma semana de tratamento, uma vez que não apresentava mais edema pulmonar na avaliação radiográfica.

Após 30 dias do início do tratamento (mar/2013), a cadela apresentou um episódio de síncope domiciliar. Foi observado pelo tutor, que era médico veterinário, que durante o episódio, o animal perdeu a consciência, apresentou vasoconstrição periférica (mucosas pálidas) e bradicardia ( $\mathrm{FC}=40$ bpm). Minutos após a crise foi realizado um ECG, o qual não revelou a presença de arritmias. Suspeitou-se de síncope por diminuição de DC ou hipertensão pulmonar, as quais foram descartadas pelo exame ecocardiográfico, uma vez que não notou-se alterações nos parâmetros de volume e na pressão pulmonar estimada pelo refluxo de tricúspide. Desta maneira, optou-se pela manutenção da terapia com enalapril, espironolactona, omega 3 e amiodarona.

Após o início do tratamento com o antiarrítmico (amiodarona), foi realizado um acompanhamento eletrocardiográfico periódico. Houve uma pequena variação no número de complexos ventriculares prematuros, sem haver um aumento significativo na sua ocorrência ou evolução para taquicardia ventricular em nenhuma das avaliações realizadas. Não foi possível a realização de Holter.

Em virtude da continuidade dos episódios de síncope (cinco episódios), optou-se pela realização do tratamento de hipertensão pulmonar com um vasodilatador arteriolar pulmonar (sildenafil ${ }^{13}$ ) (KELLIHAN et al., 2015) em outubro/2013, uma vez que os valores sugestivos de pressão pulmonar encontravam-se no limite superior na avaliação ecocardiográfica $(30 \mathrm{mmHg})$, de acordo com o cálculo realizado a partir do gradiente de regurgitação de tricúspide (CAMPBELL, 2007).

Após 30 dias do início do tratamento com sildenafil (novembro/2013), o animal apresentou nova crise de dispneia e cianose, causada por edema pulmonar, evidenciado por exame radiográfico. Os demais exames complementares foram realizados, e não apresentaram alterações significativas comparados com a avaliação anterior. A partir de então, foi instituído o tratamento contínuo com furosemida, além dos demais medicamentos já descritos inicialmente. Após a instituição da terapia com furosemida o animal apresentou apenas uma crise de síncope, permanecendo por 40 dias sem apresentar novo episódio.

Em dez/2013 o animal voltou a apresentar síncope (três episódios), e uma nova crise de dispneia e cianose. $\mathrm{O}$ animal tornou-se refratário à terapia domiciliar e foi internado para o tratamento emergencial de edema pulmonar, com aplicação parenteral de furosemida, aminofilina e butorfanol, além de oxigenioterapia. Devido à ausência de resposta ao tratamento durante dois dias, optou-se pela eutanásia do animal. Por opção do proprietário, não foi realizada a necropsia.

Segundo informações do proprietário, todos os episódios de síncope foram acompanhados por bradicardia, palidez de mucosas e precedidos por episódios de tosse, ânsia,

\footnotetext{
${ }^{8}$ Maleato de Enalapril@ comprimido 5mg, Teuto, Anápolis, GO, Brasil.

${ }^{9}$ Aldactone ${ }^{\circledR}$ comprimido $25 \mathrm{mg}$, Pfizer, Guarulhos, SP, Brasil.

${ }^{10}$ Ômega 3 cápsulas, farmácia de manipulação, Brasil.

${ }^{11}$ Ancoron ${ }^{\circledR}$ comprimido $100 \mathrm{mg}$, Libbs, São Paulo, SP, Brasil.

${ }^{12}$ Furosemida ${ }$ comprimido $40 \mathrm{mg}$, Teuto, Anápolis, GO, Brasil.

${ }^{13}$ Sildenafil cápsulas, farmácia de manipulação, Brasil.
} 
vômitos ou compressão da parede torácica por contenção física realizada pelo proprietário. Os demais exames complementares, como hemograma, perfil renal e hepático, glicemia e ultrassonografia abdominal estavam sempre normais, exceto por uma leve azotemia.

\section{Discussão}

Um diagnóstico definitivo da causa da síncope em cães é alcançado em apenas 40 a 50\% dos casos (ABBOT, 2005). Neste caso relatado, foram excluídas as causas mais comuns de síncope, sugerindo a possível presença de síncope neurocardiogênica.

A disfunção sistólica é uma das causas de síncope (DAVIDOW; PROW; WOODFIELD, 2001). No caso apresentado não houve evidências de diminuição da contratilidade cardíaca, apresentando fração de encurtamento superior a $55 \%$ em todos os exames ecocardiográficos realizados. Animais com DVCM importante que apresentem fração de encurtamento superior a $50 \%$ não apresentam disfunção miocárdica (KITLESON, 2010). Dessa forma, excluiu-se a possibilidade da má contratilidade ser a causa da síncope apresentada pelo cão.

O enchimento ventricular inadequado também poderia levar à síncope (DAVIDOW; PROW; WOODFIELD, 2001), no entanto, não houve evidências de disfunção diastólica no caso apresentado, verificado pelas medidas do Doppler pulsado no fluxo da válvula mitral (relação E/A) e Doppler tecidual (CHETBOUL, 2002; BONAGURA; SCHOBER, 2009).

A síncope também pode resultar de obstruções de fluxonas vias de saída (MOYA et al., 2009), o que não foi evidenciado no caso relatado. Obstruções do lado direito do coração, como estenose pulmonar, hipertensão pulmonar, tumores cardíacos, e dirofilariose, diminuem o retorno venoso para o lado esquerdo, reduzindo assim o enchimento ventricular e o DC. Obstruções do lado esquerdo, como estenose subaórtica, tumores intracardíacos e cardiomiopatias hipertróficas, aumentam a pós-carga e diminuem o DC (MOYA et al., 2009).

O cálculo da pressão pulmonar foi realizado por meio do gradiente de pressão da insuficiência de tricúspide e evidenciou valores no limite superior da normalidade $(30 \mathrm{mmHg})$, de acordo com valores sugeridos pela literatura (CAMPBELL, 2007). Optou-se por iniciar o tratamento com sildenafil, uma vez que é eficaz para a diminuição da pressão pulmonar por seu efeito vasodilatador arteriolar (BACH et al., 2006; KELLIHAN et al., 2015). A pressão pulmonar manteve-se estável mesmo após o início da terapia e não cessaram as crises de síncope com o uso da medicação, descartando-se a hipertensão pulmonar como a causa da síncope.

A hipotensão reduz o DC e a resistência periférica, podendo levar a síncope (DAVIDOW; PROW; WOODFIELD, 2001). Fármacos hipotensores, como os inibidores da enzima conversora da angiotensina (enalapril) também podem levar a uma diminuição do DC (ABBOT, 2008). Apesar do animal relatado ser medicado com enalapril na dose de $0,25 \mathrm{mg} / \mathrm{kg}$ a cada 12 horas, este medicamento, nesta dosagem, não foi capaz de reduzir a pressão arterial sistêmica, uma vez que o animal apresentava-se normotenso em todas as avaliações realizadas.

Estudo com 51 cães cardiopatas evidenciou que
$35 \%$ deles apresentaram síncope por algum tipo de arritmia, e o restante $(65 \%)$ por outra causa (BRIGHT; CALI, 2000). A síncope pode estar associada a intensas bradicardias (bloqueio atrioventricular de terceiro grau ou doença do nodo sinusal), que diminuem o DC por diminuir excessivamente a FC, ou taquicardias sustentadas (taquicardia atrial ou ventricular), que podem diminuir o DC por comprometer o tempo de enchimento cardíaco (DAVIDOW; PROW; WOODFIELD, 2001; JOCHMAN-EDWARDS et al., 2002; ABBOT, 2008). No caso apresentado, apesar do animal apresentar histórico de arritmia ventricular com presença de complexos ventriculares prematuros, nenhum registro eletrocardiográfico revelou taquicardia ventricular paroxística e a FC do animal era estável (100-120 bpm). Somente durante a crise de síncope revelava-se a presença de bradicardia pronunciada, assim como relatado em cães da raça Boxer que apresentavam histórico de síncope associada à bradicardia (THOMASON et al., 2008). E assim como neste relato, os cães Boxers apresentavam episódios de taquicardia ventricular mas que não estavam associados aos episódios de síncope (THOMASON et al., 2008).

A maior causa de síncope em humanos é a síncope neurocardiogênica e ocorre em cerca de $37 \%$ das pessoas ao longo da vida (GANZEBOOM et al., 2006). Este tipo de síncope refere-se a um grupo de condições em que os reflexos cardiovasculares se tornam inadequados para o controle circulatório de forma intermitente, em resposta a um estímulo, resultando em vasodilatação ou bradicardia e, assim, em uma queda na PA sistêmica e perfusão cerebral (DAVIDOW; PROW; WOODFIELD, 2001). Na medicina veterinária, foi relatado a síncope neurocardiogênica em sete cães da raça Boxers (THOMASON et al., 2008) e em cinco equinos (LYLE et al., 2010).

Exemplo comum em pessoas é a síncope em resposta a uma situação de medo ou estresse (síncope situacional). Nestes casos, o estresse conduz a um aumento do tônus simpático mediado por barorreceptores, com um aumento inicial na FC e PA. No entanto, o estresse pode também levar a uma vasodilatação muscular periférica com desvio de sangue da circulação central (DAVIDOW; PROW; WOODFIELD, 2001; MOYA et al., 2009). Este tipo de síncope também pode ocorrer em pessoas durante a postura em estação (hipotensão ortostática). A postura ereta (em vez de quadrúpede) torna as pessoas mais suscetíveis a efeitos gravitacionais sobre a circulação (GRUBB; KOSINSKI, 2001). Em ambas as situações, contrações vigorosas de um ventrículo com pouco volume podem levar a estimulação de mecanorreceptores, vasodilatação paradoxal e bradicardia (MOYA et al., 2009;DAVIDOW; PROW; WOODFIELD, 2001; GRUBB; KOSINSKI, 2001).

Apesar de episódios de síncope emocional ou relacionada ao estresse não serem documentados em animais, a síncope após a tosse - um tipo de síncope vasovagal situacional - é bastante comum em raças pequenas e braquicefálicas (ABBOT, 2008). A tosse aumenta a pressão intratorácica e a pressão intracraniana transitoriamente. $\mathrm{O}$ aumento da pressão intratorácica diminui o retorno venoso para o coração e leva à redução do DC quando a pressão intracraniana está alta. Isto pode resultar em síncope secundária à baixa perfusão cerebral. A tosse também pode estimular o nervo vago nas vias aéreas superiores e causar bradicardia, vasodilatação e colapso (MAZNYCZKA; SAQUIRE, 2010). A síncope por 
tosse em cães é frequentemente associada com o colapso de traquéia, doença pulmonar obstrutiva crônica e DVCM com aumento atrial (KITTLESON, 2010). No atual caso, o colapso traqueal foi excluído por meio da avaliação das imagens radiográficas.

O diagnóstico de síncope neurocardiogênica em pessoas podem incluir testes de inclinação e um teste provocativo de estresse ortostático. A utilidade potencial destas técnicas em medicina veterinária é desconhecida (SCHNIPPER; KAPOOR, 2001).

\section{Conclusão}

No caso relatado as crises de síncope foram sempre relacionadas com bradicardia e aumento da pressão intratorácica, como nos casos de tosse, ânsia ou episódios eméticos, pressão manual exercida pelo proprietário ao segurar/levantar o animal. Dessa forma, excluindo-se as demais causas de síncope cardíaca, associado aos sinais clínicos apresentados durante os episódios e aos fatores associados com aumento da pressão intratorácica, pressupõe-se que a síncope apresentada pelo animal fosse do tipo neurocardiogênica. Este relato torna-se importante para alertar o clínico para mais uma causa de síncope em cães e, dessa forma, tentar evitar os fatores que desencadeiam os episódios de síncope como uma forma de prevenção e melhoria do bem-estar dos animais acometidos.

\section{Referências}

AZEVEDO, M. C; BARBISAN, J. N.; SILVA, E. O. Neurocardiogenic syncope and hereditarity. Revista da Associação Médica Brasileira, v. 55, n. 1, p. 19-21, 2009.

ABBOT, J. A. Acquired valvular disease. In: TILLEY, L. P. (Ed.) Manual of canine and feline cardiology. Philadelphia: Saunders, 2008. p.78-98.

BACH, J. F. et al. Retrospective evaluation of sildenafil citrate as a therapy for pulmonary hypertension in dogs. Journal of Veterinary Internal Medicine, v. 20, n. 5, p. 1132-1135, 2006.

BONAGURA, J. D.; SCHOBER, K. E. Can ventricular function be assessed by echocardiography in chronic canine mitral valve disease? Journal of Small Animal Practice,v. 50, n. 1, p. 12-24, 2009.

BRIGHT, J. M.; CALI, J. V. Clinical usefulness of cardiac event recording in dogs and cats examined because of syncope, episodic collapse, or intermittent weakness: 60 cases (1997-1999). Journal of the American Veterinary Medical Association, v. 216, n. 7, p. 1110-1114, 2000.

CAMPBELL, F. E. Cardiac effects of pulmonary disease. Veterinary Clinics of North American -Small Animal Practice, v. 37, n. 5, p. 949-962, 2007.

CHETBOUL, V. Tissue doppler imaging: a promising technique for quantifying regional myocardial function.

Journal of Veterinary Cardiology, v. 4, n. 2, p.7-12, 2002.
DAVIDOW, E. B.; PROW, L. X.; WOODFIELD, J. A. Syncope: pathophysiology and differential diagnosis.

Compendium of Continuing Education Practice, v. 23, n. 7,p. 608-620, 2001.

DeFRANCESCO, T. C. Management of cardiac emergencies in small animals. Veterinary Clinics of Small Animal, v. 43, n. 4, p. 817-842, 2013.

GANZEBOOM, K.S. et al. Lifetime cumulative incidence of syncope in the general population: a study of 549 Dutch subjects aged 35-60 years. Journal of Cardiovascular Electrophysiology, v. 17, n. 11, p. 1172-1176, 2006.

GRUBB, B. P.; KOSINSKI, D. J. Syncope resulting from autonomic insufficiency syndromes associated with orthostatic intolerance. Medical Clinics of North America, v. 85, n. 2, p. 457-472, 2001.

JOCHMAN-EDWARDS, C. M. et al. Electrocardiographic findings in miniature schnauzers with syncope. Journal of Veterinary Emergency and Critical Care, v. 2, n. 4, p. 253-259, 2002.

KELLIHAN, H. B. et al. Acute resolution of pulmonary alveolar infiltrates in 10 dogs with pulmonary hypertension treated with sildenafil citrate: $2005 \mathrm{e} 2014$. Journal of Veterinary Cardiology, v. 17, n. 3, p. 182-191, 2015.

KITTLESON, M. D. Myxomatous atrioventricular valvular degeneration. In: KITTLESON, M. D.; KIENLE, R. D. (Ed.) Small animal cardiovascular medicine, St. Louis:Editora Mosby, 1998. p.297-318.

LYLE, C.H. et al. Retrospective evaluation of episodic collapse in the horse in a referred population: 25 cases (1995-2009). Journal of Veterinary Internal Medicine, v. 24, n. 6, p. 1498-1502, 2010.

MAZNYCZKA, A.; SQUIRE, I. B. Cough syncope - a diagnosis under pressure. Lancet, v. 376, n. 9739, p. 486, 2010 .

MOYA, A. et al. Guidelines for the diagnosis and management of syncope.European Heart Journal, v. 25, n. 5, p. 2631-2671, 2009.

SCHNIPPER, J. L.; KAPOOR, W. N. Diagnostic evaluation and management of patients with syncope. Medical Clinics of North America,v. 85, n. 2, p. 423-456, 2001.

THOMASON, J. D. et al. Bradycardia-associated syncope in 7 boxers with ventricular tachycardia (2002-2005). Journal of Veterinary Internal Medicine, v. 22, n. 4, p. 931-936, 2008.

Recebido em: 07.05.2015 Aceito em: 01.12.2016 